\title{
THE ROLE OF AN INTEGRATION IDENTITY IN THE ANALYSIS OF THE CAUCHY-LERAY TRANSFORM
}

\author{
LOREDANA LANZANI* AND ELIAS M. STEIN*
}

\begin{abstract}
The purpose of this paper is to complement the results in LS-1 by showing the dense definability of the Cauchy-Leray transform for the domains that give the counterexamples of [LS-1], where $L^{p}$-boundedness is shown to fail when either the "near" $C^{2}$ boundary regularity, or the strong $\mathbb{C}$-linear convexity assumption is dropped.
\end{abstract}

\section{Dedicated to the memory of Professor Minde Cheng on the occasion of the centenary of his birth}

\section{INTRODUCTION}

The purpose of this paper is to complement the previous results in [LS-1] and [LS-2] which deal with the $L^{p}$-boundedness of the CauchyLeray integral in $\mathbb{C}^{n}, n \geq 2$, proved under optimal regularity assumptions and geometric restrictions.

If $D$ is a suitable (convex) domain in $\mathbb{C}^{n}$ then we can define the Cauchy-Leray integral [LS-2] $\mathbf{C}(f)$ for an appropriate function $f$ given on the boundary $b D$ of $D$. The integral $\mathbf{C}(f)(z)$ is defined for $z \in D$ and has the following properties: first, $\mathbf{C}(f)(z)$ is always holomorphic for $z \in D$; and second, if $f=\left.F\right|_{b D}$ where $F$ is continuous in $\bar{D}$ and holomorphic in $D$, then $\mathbf{C}$ reproduces $f$, i.e. $\mathbf{C}(f)(z)=F(z)$, for $z \in D$.

The well-known theory of the Cauchy integral in $\mathbb{C}$ (see $[\mathrm{C}$, [CMM], [Da] and in particular the classical theorem of M. Riesz for the unit disc, raise the question of the corresponding $L^{p}$-boundedness of the

* This material is based upon work supported in part by the National Science Foundation under awards No. DMS-1503612 (Lanzani) and DMS-1265524 (Stein), while both authors were in residence at the Mathematical Sciences Research Institute in Berkeley, California, during the Spring 2017 semester.

2000 Mathematics Subject Classification: 30E20, 31A10, 32A26, 32A25, 32A50, 32A55, 42B20, 46E22, 47B34, 31B10.

Keywords: Hardy space; Cauchy Integral; Cauchy-Szegő projection; Lebesgue space; pseudoconvex domain; minimal smoothness; Leray-Levi measure. 
Cauchy-Leray integral for $n \geq 2$, and more particularly determining the optimal conditions both on the regularity of the domain $D$ and the nature of the convexity of $D$, for which this boundedness holds. To be precise, suppose $f$ is a $C^{1}$ function on the boundary of $D$, then under the circumstances detailed below we can define the induced "CauchyLeray transform" of $f, \mathcal{C}(f)$, as a function on the boundary of $D$ given by

$$
\mathcal{C}(f)=\left.\mathbf{C}(f)\right|_{b D}
$$

and it is proved in [LS-2] that the mapping $f \mapsto \mathcal{C}(f)$ extends to a bounded transformation on $L^{p}(b D, d \sigma), 1<p<\infty$, where $d \sigma$ is the induced Lebesgue measure on $b D$. This assertion holds under the following two conditions:

(i) The boundary has regularity "near" $C^{2}$, in the sense that $b D$ is of class $C^{1,1}$.

(ii) The boundary is "strongly $\mathbb{C}$-linearly convex".

The convexity condition (ii) is weaker than strong convexity (the strict positive-definiteness of the real quadratic fundamental form at each boundary point); however it is stronger than strong-pseudoconvexity (the strict positivity of the Levi form).

In [LS-1] two simple counter-examples, elucidating the necessary nature of both conditions were given. These are in terms of two elementary domains in $\mathbb{C}^{2}$. With $z=\left(z_{1}, z_{2}\right)$, and $z_{1}=x_{1}+i y_{1}$ they are given by

$$
\left\{z:\left|z_{2}\right|^{2}+x_{1}^{2}+y_{1}^{4}<1\right\}
$$

and

$$
\left\{z:\left|z_{2}\right|^{2}+\left|x_{1}\right|^{m}+y_{1}^{2}<1\right\} \quad \text { where } \quad 1<m<2 .
$$

Equivalently, we could replace $z_{2}$ by $z_{2}-i$, as we did in LS-1]. The first domain (2) has a $C^{\infty}$ (in fact real-analytic) boundary, is strongly pseudo-convex but not strongly $\mathbb{C}$-linearly convex. The second domain (3) is of class $C^{2-\epsilon}$, with $\epsilon=2-m$, but on the other hand, is strongly $\mathbb{C}$-linearly convex.

For both domains we proved in [LS-1] that $L^{p}$-boundedness failed for all $p, 1 \leq p \leq \infty$, in the following sense. Whenever $f$ is a bounded 
function on $b D$ supported on a proper subset of the boundary, $\mathbf{C}(f)(z)$ can be defined (as an absolutely convergent integral) whenever $z \in b D$ is at a positive distance from the support of $f$, (which we still denote by $\mathbf{C}(f)(z))$. One might ask if there is an inequality of the form

$$
\|\mathbf{C}(f)\|_{L^{p}(S)} \leq A_{p}\|f\|_{L^{p}(b D)}
$$

whenever $S$ is a subset of $b D$ disjoint from the support of $f$, with the bound in (44) independent of $f$ or $S$, and with the underlying measure the induced Lebesgue measure. This was shown in [LS-1] to fail for both domains for all $p, 1 \leq p \leq \infty$.

In order to define the Cauchy-Leray transform for the domains that satisfy (i) and (ii), it was shown in [LS-2] that whenever $f$ is of class $C^{1}$ on the boundary, then $\mathbf{C}(f)(z)$ extends to a continuous function on $\bar{D}$. It is our purpose here to demostrate that a similar assertion holds for the counter-example domains (2) and (3) and thus the induced Cauchy-Leray transform $f \mapsto \mathcal{C}(f)$, initially defined for $C^{1}$-functions, does not extend to a bounded operator on $L^{p}$, for any $p$, for both domains (2) and (3).

Restricting ourselves to the case $n=2$, one can write the CauchyLeray integral as

$$
\mathbf{C}(f)(z)=\int_{w \in b D} \frac{f(w)}{(\Delta(w, z))^{2}} d \lambda(w)
$$

where $\Delta(w, z)$ is the non-hermitian pairing

$$
\langle\partial \rho(w), w-z\rangle=\sum_{j=1}^{2} \frac{\partial \rho(w)}{\partial w_{j}}\left(w_{j}-z_{j}\right),
$$

with $\rho$ a defining function of $D$, and $d \lambda$ the corresponding Leray-Levi measure.

For the domains that satisfy (i) and (ii), the continuity of $\mathbf{C}(f)(z)$, $z \in \bar{D}$ when $f$ is of class $C^{1}$ on $b D$ can be shown as a consequence of the principle that the Cauchy-Leray kernel (when $n \geq 2$ ) is a "derivative". This is most aptly expressed in the global integration-by-parts performed in the proof of Proposition 5 below, which can be summarized as

$$
\mathbf{C}(f)=\mathbf{E}(d f)+\mathbf{R}(f)+f
$$

Here the kernels of the operators $\mathbf{E}$ and $\mathbf{R}$ both have singularities weaker than that of $\mathbf{C}$ and are in fact absolutely integrable singularities. The one for $\mathbf{E}$ is of the order 


$$
\frac{1}{|\Delta(w, z)|}
$$

and the one for $\mathbf{R}$ is of the order

$$
\frac{|z-w|}{|\Delta(w, z)|^{2}}, \quad \text { or even } \quad \frac{|z-w|^{2}}{|\Delta(w, z)|^{2}} .
$$

However application of this formalism to the first example, (2), is problematic: because of the "flatness" of that domain (which can be restated in terms of the higher degree of vanishing of $\Delta(w, z)$ along the diagonal $\{w=z\}$, see (9)), the integrability of (8) cannot be guaranteed because the numerator in (8) does not help to control the singularities that are away from the diagonal. Thus in this case a different argument is needed, one that uses a local integration by parts, depending on the location of the coordinate patch with respect to the "flat" part of the boundary. This is carried out in Proposition 2 in Section 2 below.

In the second example the difficulty comes from the lack of $C^{2}$ regularity. Here, however, a simple but critical modification of the argument that was used in [LS-2] to prove an earlier version of (6) for the "nice "domains works for the most relevant choices of $m$ that pertain the domain (3). This is carried out in Proposition 5 and Lemma 6 in Section 3 below.

These results together with what was done in LS-2] then give us our main conclusion:

Theorem 1. In the cases of the domain (2), and the domain (3) for $3 / 2<m<2$, for any function $f$ of class $C^{1}$ on $b D$, the Cauchy integral $\mathbf{C}(f)(z)$ extends to a continuous function on $\bar{D}$. If we set

$$
\mathcal{C}(f)=\left.\mathbf{C}(f)\right|_{b D}
$$

then the mapping $f \mapsto \mathcal{C}(f)$ cannot be extended to a bounded operator on $L^{p}(b D)$ for any $p, 1 \leq p \leq \infty$.

There are two additional comments to make. First, since the main interest of the second example is the class $C^{2-\epsilon}$ with $\epsilon$ small, this clearly falls within the restriction $3 / 2<m<2$. Second, there is a weaker conclusion that covers the full range $1<m<2$. This is stated in Proposition 7 below. 


\section{THE FIRST EXAMPLE}

Writing $z_{j}=x_{j}+i y_{j}$ for $j=1,2$, our first example is the domain (2), that is

$$
D=\left\{\left(z_{1}, z_{2}\right):\left|z_{2}\right|^{2}+x_{1}^{2}+y_{1}^{4}<1\right\}
$$

2.1. The Cauchy-Leray integral for the domain (2). Recall that $D$ is strictly convex and this grants

$$
\begin{gathered}
(\partial \rho(w), w-z)_{\mathbb{R}} \geq \\
\geq\left(x_{1}-u_{1}\right)^{2}+\left(x_{2}-u_{2}\right)^{2}+\left(y_{2}-v_{2}\right)^{2}+\left(v_{1}^{2}+y_{1}^{2}\right)\left(v_{1}-y_{1}\right)^{2}
\end{gathered}
$$

when $w, z \in b D$. From this strict convexity it follows that $D$ supports the Cauchy-Leray integral

$$
\mathbf{C} f(z)=\frac{1}{(2 \pi i)^{2}} \int_{w \in b D} f(w) j^{*} \frac{(\partial \rho \wedge \bar{\partial} \partial \rho)(w)}{\langle\partial \rho(w), w-z\rangle^{2}}, \quad z \in D,
$$

where $j^{*}$ denotes the pullback via the inclusion $j: b D \hookrightarrow \mathbb{C}^{2}$, and in fact with respect to the induced Lebesgue measure $d \sigma$, we have

$$
\mathbf{C} f(z)=\int_{w \in b D} \frac{f(w) \Gamma(w)}{\langle\partial \rho(w), w-z\rangle^{2}} d \sigma(w), \quad z \in D,
$$

with $\Gamma$ a smooth, strictly positive function on $b D$, see [LS-1].

Proposition 2. Suppose that $f \in C^{1}(b D)$. Then $\mathbf{C} f(z)$ extends to a continuous function on $\bar{D}$.

This proposition allows us to define the induced Cauchy-Leray transform $\mathcal{C}(f)$, at least initially for $f$ that are of class $C^{1}$ on $b D$, by

$$
\mathcal{C} f=\left.\mathbf{C} f\right|_{b D} .
$$

2.2. Proof of Proposition 2. Throughout this section we shall simplify the notation slightly by writing $\rho$ instead of $\rho$, and by setting

$$
\Delta(w, z)=\langle\partial \rho(w), w-z\rangle .
$$

We need first a local representation of the Cauchy-Leray integral, which was the idea that $\Delta(w, z)$ arises as a "derivative".

We fix a point $\zeta \in b D$, and we will restrict attention to points $z \in \bar{D}, w \in b D$ both near $\zeta$. We can introduce a new coordinate system, centered at $\zeta$, so that we pass from the original coordinates in $\mathbb{C}^{2}$ to the present coordinates by a translation and a unitary linear 
transformation, and so that in the new coordinates $w=\left(w_{1}, w_{2}\right), w_{j}=$ $u_{j}+i v_{j}$, the point $\zeta$ has coordinates $(0,0)$ and

$$
\frac{\partial \rho}{\partial v_{2}}(\zeta)=|\nabla \rho(\zeta)|, \text { while } \frac{\partial \rho}{\partial u_{1}}(\zeta)=\frac{\partial \rho}{\partial v_{1}}(\zeta)=\frac{\partial \rho}{\partial u_{2}}(\zeta)=0 .
$$

Since then the tangent space to $b D$ at $\zeta$ is given by $\left\{\left(u_{1}+i v_{1}, u_{2}\right)\right\}$ we can express $b D$ near $\zeta$ as a graph $v_{2}=\Phi\left(u_{1}, v_{1}, u_{2}\right)$, with $\Phi(0,0,0)=0$ and $\nabla \Phi(0,0,0)=(0,0,0)$. In particular if $w=\left(w_{1}, w_{2}\right) \in b D$ is close to $\zeta$ with say, $|w-\zeta|<\delta$ then

$$
\frac{\partial w_{2}}{\partial u_{2}}=1+i \frac{\partial \Phi}{\partial u_{2}}=1+i O(\delta) .
$$

Lemma 3. We have

$$
\frac{\partial \Delta}{\partial u_{2}}(w, z) \neq 0, \text { if }|w-\zeta|<\delta \text {, and }|z-\zeta|<\delta
$$

when $\delta$ is sufficiently small.

Proof.

$$
\frac{\partial \Delta}{\partial u_{2}}(w, z)=I+I I+I I I
$$

where

$$
I=\left\langle\frac{\partial}{\partial u_{2}} \partial \rho(w), w-z\right\rangle
$$

and thus $|I| \leq c \delta$ because $|w-z| \leq 2 \delta$; and

$$
I I=\left\langle\partial \rho(w)-\partial \rho(\zeta), \frac{\partial w}{\partial u_{2}}\right\rangle
$$

and therefore $|I I| \leq c \delta$ because $|w-\zeta| \leq \delta$. However

$$
\begin{gathered}
I I I=\left\langle\partial \rho(\zeta), \frac{\partial w}{\partial u_{2}}\right\rangle=\left[0, \frac{1}{2}\left(\frac{\partial \rho}{\partial u_{2}}(\zeta)-i \frac{\partial \rho}{\partial v_{2}}(\zeta)\right)\right] \cdot[0,(1+i O(\delta))]= \\
=-i|\nabla \rho(\zeta)|(1+i O(\delta))=-i \frac{|\nabla \rho(\zeta)|}{2}+O(\delta) .
\end{gathered}
$$

Thus $|I I I| \geq|\nabla \rho(\zeta)|>c_{1}>0$ and so (12) is established.

For $w$ in the above neighbrhood of $\zeta$, let us write

$$
\Gamma(w) d \sigma(w)=\Lambda\left(u_{1}, v_{1}, u_{2}\right) d u_{1} d v_{1} d u_{2}, \quad \text { and } \quad \gamma(w)=\frac{\Lambda(w)}{\frac{\partial \Delta}{\partial u_{2}}(w, z)} .
$$

Then $\gamma(w)$ is a smooth function (uniformly in $z$ for $|z-\zeta|<\delta$ ). 
Corollary 4. Suppose $f$ is a $C^{1}$ function on bD supported in the above neighborhood of $\zeta$. If $|z-\zeta|<\delta, z \in D$, then

$$
\mathbf{C} f(z)=\int_{b D} \frac{1}{\Delta(w, z)} \frac{\partial}{\partial u_{2}}(f(w) \cdot \gamma(w)) d u_{1} d v_{1} d u_{2} .
$$

Proof.

$$
\mathbf{C} f(z)=\int_{b D} \frac{1}{\Delta^{2}(w, z)} f(w) \Gamma(w) d \sigma(w)
$$

Then in the support of $f$, we have

$$
\frac{1}{\Delta^{2}(w, z)}=-\frac{1}{\frac{\partial \Delta}{\partial u_{2}}(w, z)} \cdot \frac{\partial}{\partial u_{2}}\left(\frac{1}{\Delta(w, z)}\right) .
$$

Inserting this in the above and carrying out the indicated integration by parts in the $u_{2}$ variable then yields (13).

To continue we make several other observations.

First, whenever $z \in b D$, write $z_{\epsilon}=z+\epsilon \nu(z)$, where $\nu(z)$ is the inward unit normal vector at $z$ and $\epsilon \geq 0$, that is

$$
\nu(z)=-\frac{\nabla \rho(z)}{|\nabla \rho(z)|} .
$$

Assume as before that $w, z \in b D,|z-w| \leq 2 \delta$. Then with $\delta$ sufficiently small

$$
\operatorname{Re} \Delta\left(w, z_{\epsilon}\right) \geq c \epsilon+\operatorname{Re} \Delta(w, z) \text { with } c>0 .
$$

In fact

$$
\Delta\left(w, z_{\epsilon}\right)=\Delta(w, z)-\epsilon\langle\partial \rho(w), \nu(z)\rangle .
$$

But $\nu(z)=\nu(w)+O(|z-w|)$, thus

$$
\langle\partial \rho(w), \nu(z)\rangle=\langle\partial \rho(w), \nu(w)\rangle+O(|z-w|),
$$

and altogether

$$
\Delta\left(w, z_{\epsilon}\right)-\Delta(w, z)=-\epsilon\langle\partial \rho(w), \nu(w)\rangle+O(\epsilon|z-w|) .
$$

Since $\operatorname{Re}\langle\partial \rho(w), \nu(w)\rangle=-|\nabla \rho(w)|$, this yields (14).

In fact, if $|w-z|>2 \delta$ then the strict convexity of $D$ gives that $\operatorname{Re} \Delta\left(w, z_{\epsilon}\right)>\widetilde{c}$; by the compactness of $\bar{D}$ if we take $0<c_{0}<1$ sufficiently small, we may combine all of the above and conclude that

$$
\operatorname{Re} \Delta\left(w, z_{\epsilon}\right) \geq c_{0}(\epsilon+\operatorname{Re} \Delta(w, z)) \text { uniformly in } z, w \in b D \text {. }
$$


The key observation is that

$$
\int_{b D} \frac{1}{(\operatorname{Re} \Delta(w, z))^{1+\beta}} d \sigma(w) \leq C_{\beta}
$$

uniformly for $z \in b D$, if $0 \leq \beta<1 / 4$, and with $\sigma$ the induced Lebesgue measure on $b D$.

For small fixed $\eta>0$, we may assume that the integration in (17) is over the $\eta$-ball centered at $z$; otherwise the strict convexity of $D$ gives that $\operatorname{Re} \Delta(w, z) \geq c>0$ if $|w-z| \geq \eta$, and the compactness of $\bar{D}$ grant that the inequality (17) holds when the integration is taken over the complement of the $\eta$-ball about $z$.

To illustrate what is done next, assume that $z$ lies in the ball centered at the origin. Then in the coordinates $z=\left(z_{1}, z_{2}\right)$ and $w=\left(w_{1}, w_{2}\right)$, we know by (9) that in particular

$$
2 \operatorname{Re} \Delta(w, z) \geq\left(x_{1}-u_{1}\right)^{2}+\left(x_{2}-u_{2}\right)^{2}+v_{1}^{2}\left(v_{1}-y_{1}\right)^{2} .
$$

Moreover the tangent space to $b D$ at the origin is given by $\left(u_{1}+\right.$ $\left.i v_{1}, u_{2}\right)$. So far $w$ is near the origin, $b D$ is represented by a graph $\left(u_{1}+i v_{1}, u_{2}+i \Phi\left(u_{1}, v_{1}, u_{2}\right)\right)$ with $\Phi$ a smooth function, and hence the induced measure $d \sigma$ is given by $d \sigma=D\left(u_{1}, v_{1}, u_{2}\right) d u_{1} d v_{1} d u_{2}$, where the density $D$ is bounded. This shows that (17) will be proved as soon as we have that

$$
\int_{B} \frac{1}{\left(u_{1}^{2}+u_{2}^{2}+v_{1}^{2}\left(v_{1}-y_{1}\right)^{2}\right)^{1+\beta}} d u_{1} d u_{2} d v_{1}<\infty \text { uniformly in } y_{1}
$$

where $B$ is the unit ball: $\left\{u_{1}^{2}+v_{1}^{2}+u_{2}^{2}<1\right\}$ in the parameter space.

However

$$
\int_{\mathbb{R}^{2}} \frac{1}{\left(u_{1}^{2}+u_{2}^{2}+A\right)^{1+\beta}} d u_{1} d u_{2}=C_{\beta} A^{-\beta}, A>0, \beta>0
$$

as a simple rescaling $u_{1}=u_{1}^{\prime} A^{1 / 2}, u_{2}=u_{2}^{\prime} A^{1 / 2}$ shows. So we take $A=\left(v_{1}\left(v_{1}-y_{1}\right)\right)^{2}$, and observe that

$$
\int_{\left|v_{1}\right| \leq 1} \frac{1}{\left|v_{1}\right|^{2 \beta}\left|v_{1}-y_{1}\right|^{2 \beta}} d v_{1}<\infty, \text { if } 0 \leq \beta<1 / 4
$$

and this proves (18) for $0<\beta<1 / 4$ and thus also for $\beta=0$.

We next lift the restriction that $z$ lies in the $\eta$-neighborhood of the origin, and assume instead that $z$ lies in the $\eta$-neighborhood of $\zeta$, for some fixed $\zeta \in b D$. Now the tangent space to $b D$ at $\zeta$ is given by $\left\{w:(\nu(\zeta), w-\zeta)_{\mathbb{R}}=0\right\}$, where $\nu(\zeta)$ is the inner unit normal at $\zeta$. 
We consider the four real component of $\nu(\zeta)$, that we list as $\nu_{1}, \nu_{1}^{\prime}, \nu_{2}, \nu_{2}^{\prime}$, which correspond to the the $u_{1}, v_{1}, u_{2}, v_{2}$ variables, respectively. Denote by $\bar{\nu}$ a component among these four that has the maximum absolute value. Now there are two cases: Case 1: $\bar{\nu} \neq \nu_{1}^{\prime}$; Case 2: $\bar{\nu}=\nu_{1}^{\prime}$.

Now in Case 1 (which is what happens at $\zeta=0$, since there $\bar{\nu}=$ $\left.\nu_{2}=1\right)$, assume momentarily that $\bar{\nu}=\nu_{1}$. Then the tangent space at $\zeta=\left(\xi_{1}+i \eta_{1}, \xi_{2}+i \eta_{2}\right)$ can be written as

$$
u_{1}-\xi_{1}=a\left(v_{1}-\eta_{1}\right)+b\left(u_{2}-\xi_{2}\right)+c\left(v_{2}-\eta_{2}\right)
$$

with $|a|,|b|,|c| \leq 1$. So if we take $v_{1}, u_{2}$ and $v_{2}$ as independent variables to represent $b D$ as a graph, we see by (9) that it suffices to show that

$$
\int_{B^{\prime}} \frac{1}{\left(v_{2}^{2}+u_{2}^{2}+v_{1}^{2}\left(v_{1}-y_{1}\right)^{2}\right)^{1+\beta}} d v_{2} d u_{2} d v_{1}<\infty \text { uniformly in } y_{1},
$$

which is the same as (18), except that $u_{1}$ has been replaced by $v_{2}$. Similarly if $\bar{\nu}=\nu_{2}$, or $\bar{\nu}=\nu_{2}^{\prime}$.

Now in Case 2, when $\bar{\nu}=\nu_{1}^{\prime}$, we represent the tangent space as

$$
v_{2}-\eta_{2}=a^{\prime}\left(u_{1}-\xi_{1}\right)+b^{\prime}\left(u_{2}-\xi_{2}\right)+c^{\prime}\left(v_{2}-\eta_{2}\right)
$$

and then by (9) it suffices to see that

$$
\int_{B^{\prime}} \frac{1}{\left(u_{1}^{2}+u_{2}^{2}+v_{2}^{2}\right)^{1+\beta}} d u_{1} d u_{2} d v_{2}<\infty
$$

which in fact holds for $0 \leq \beta<1$. This concludes the proof of (17).

Returning to the proof of Proposition 2, we will show that whenever $f$ is a $C^{1}$ function on $b D$, then $\mathbf{C} f\left(z_{\epsilon}\right)$ converges uniformly for $z \in b D$, as $\epsilon \rightarrow 0$. To see this we decompose $f$ as a finite sum

$$
f=\sum_{j=1}^{N} f_{j}
$$

where each $f_{j}$ is a $C^{1}$ function supported in a ball $B_{j}$ of radius $\eta / 2$, centered at $\zeta^{(j)} \in b D$. If $z \in B_{j}^{*}$ (the ball with the same center but twice the radius) and $\eta$ is sufficiently small, then by Corollary 4

$$
\mathbf{C} f_{j}\left(z_{\epsilon}\right)=\int_{b D} \frac{1}{\Delta\left(w, z_{\epsilon}\right)} \frac{\partial}{\partial u_{2}}\left(f_{j} \gamma\right) d u_{1} d v_{1} d u_{2}
$$

Next observe that

$$
\int_{b D}\left|\frac{1}{\Delta\left(w, z_{\epsilon}\right)}-\frac{1}{\Delta(w, z)}\right| d \sigma(w) \lesssim \epsilon^{\beta}
$$


Indeed because both $\operatorname{Re} \Delta\left(w, z_{\epsilon}\right)$ and $\operatorname{Re} \Delta(w, z)$ are non-negative and $\Delta\left(w, z_{\epsilon}\right)-\Delta(w, z)=O(\epsilon)$, see (15), the integrand in (20) is dominated by

$$
\frac{c \epsilon}{\operatorname{Re} \Delta\left(w, z_{\epsilon}\right) \operatorname{Re} \Delta(w, z)} .
$$

But (15) tells us more precisely that

$$
\operatorname{Re} \Delta\left(w, z_{\epsilon}\right) \geq c_{0} \operatorname{Re} \Delta(w, z) \text { and } \operatorname{Re} \Delta\left(w, z_{\epsilon}\right) \geq c_{0} \epsilon .
$$

As a result, the integrand in (20) is dominated by a multiple of

$$
\frac{\epsilon^{\beta}}{(\operatorname{Re} \Delta(w, z))^{1+\beta}},
$$

and we need only invoke (17) to get (20). From this and (19) we obtain that $\mathbf{C} f_{j}\left(z_{\epsilon}\right)$ converges uniformly for $z \in B_{j}^{*}$ as $\epsilon \rightarrow 0$. However when $z \notin B_{j}^{*}$, then $\Delta\left(w, z_{\epsilon}\right) \neq 0$ for the relevant $w$ and $z$, and the convergence as $\epsilon \rightarrow 0$ is obvious. This gives the desired result for each $f_{j}$, and hence for their sum, proving Proposition 2.

\section{THE SECOND EXAMPLE}

For our second example, the domain (3), we choose

$$
\rho(w)=\left|w_{2}\right|^{2}+\left|u_{1}\right|^{m}+v_{1}^{2}-1
$$

as a defining function. Here and in the sequel we make use of the notation:

$$
\left[u_{1}\right]=\left|u_{1}\right|^{m-1} \operatorname{sign}\left(u_{1}\right) .
$$

The Cauchy-Leray denominator $\Delta(w, z)=\langle\partial \rho(w), w-z\rangle$ for $D$ is then

$$
\begin{gathered}
\Delta(w, z)= \\
\frac{1}{2}\left(m\left[u_{1}\right]^{m-1}+2 i+2 v_{1}\right)\left(u_{1}-v_{1}+i\left(v_{1}-y_{1}\right)\right)+\left(u_{2}-i v_{2}\right)\left(u_{2}-x_{2}+i\left(v_{2}-y_{2}\right)\right) .
\end{gathered}
$$

As a preliminary step we decompose the boundary of $D$ into finitely many coordinate patches, either of the first or the second kind. The coordinate patches of the first kind are centered at points which lie in the critical variety (where $u_{1}=0$ ). Those of the second kind are at a positive distance from that variety. We then decompose our given $C^{1}$-function $f$ as a sum of $C^{1}$ functions, each supported on one of these patches. For those of the second type, since we are now where matters are regular, we may argue as in Section 2. This reduces matters to the patches of the first kind. Since for these patches we have that $u_{1}$ is small, then one of the three variables $v_{1}, u_{2}, v_{2}$ must be bounded away from zero. Whichever is can be taken as the dependent variable in 
the representation of $b D$ as a graph over that patch. For simplicity of notation here we assume it is $v_{1}$, but if it were $u_{2}$ or $v_{2}$ instead, then the argument below would be unchanged. Then for $v_{1}$ (the dependent variable) we have

$$
v_{1}=\left(1-\left(\left|u_{1}\right|^{m}+\left|w_{2}\right|^{2}\right)\right)^{1 / 2} \approx 1-\frac{1}{2}\left(\left|u_{1}\right|^{m}+\left|w_{2}\right|^{2}\right) .
$$

The following basic estimates for $\Delta(w, z)$ can be proved as in LS-2, Lemma 4.3]:

$$
\operatorname{Re} \Delta\left(w, z_{\epsilon}\right)-\operatorname{Re} \Delta(w, z) \gtrsim \epsilon
$$

where as before, $z_{\epsilon}=z+\epsilon \nu(z)$, and

$$
\left|\Delta\left(w, z_{\epsilon}\right)-\Delta(w, z)\right| \lesssim \epsilon
$$

for any $w, z \in b D$.

3.1. The Cauchy-Leray integral for the domain the domain (3).

Suppose that $f \in C^{1}(b D)$ and set

$$
\mathbf{C} f(z)=\int_{w \in b D} \frac{f(w)}{\Delta^{2}(w, z)} d \lambda(w) \quad z \in D
$$

with $d \lambda(w)=j^{*}(\partial \rho(w) \wedge \bar{\partial} \partial \rho(w))$. Then in fact

$$
\mathbf{C}(f)(z)=\int_{w \in b D} f(w) j^{*}(\eta(w, z) \wedge \bar{\partial} \eta(w, z))
$$

where

$$
\eta(w, z):=\frac{\partial \rho(w)}{\Delta(w, z)}
$$

is a so-called generating form for D, see [LS-3, Sections 4.1 and 9.2]. The Cauchy-Fantappiè theory then grants that

$$
\mathbf{C}(g)(z)=g(z), \quad z \in D
$$

whenever $g$ is holomorphic in $D$ and continuous on $\bar{D}$, see [LS-3, Section 5]. In particular choosing $g(z)=1$ (the constant 1 ) we have that

$$
\mathbf{C}(1)(z)=1 \quad \text { for } z \in D \text { and thus for } z \in \bar{D}
$$

and from this it follows that

$$
\mathbf{C}(f)(z)=\int_{w \in b D} \frac{f(w)-f(z)}{\Delta^{2}(w, z)} d \lambda(w)+f(z), \quad z \in D .
$$


Proposition 5. Let $D$ be the domain (3) with $3 / 2<m<2$. Suppose that $f \in C^{1}(b D)$. Then $\mathbf{C}(f)$ extends to a continuous function in $\bar{D}$. More precisely, for $z \in b D$ set

$$
z_{\epsilon}=z+\epsilon \nu(z) \in D \text {. }
$$

Then, we have that $\mathbf{C}(f)\left(z_{\epsilon}\right)$ converges uniformly in $z \in b D$ to a limit that we denote $\mathcal{C}(f)(z)$. Furthermore, we have that such limit admits the representation

$$
\mathcal{C}(f)(z)=\mathbf{E}(d f)(z)+\mathbf{R}(f)(z)+f(z), \quad z \in b D
$$

where $\mathbf{E}(d f)$ and $\mathbf{R}(f)$ are absolutely convergent integarls given explicitly by (28) and (29) below.

Proof. By the above considerations we have that

$$
\mathbf{C}(f)\left(z_{\epsilon}\right)=\int_{w \in b D} \frac{f(w)-f(z)}{\Delta^{2}\left(w, z_{\epsilon}\right)} d \lambda(w)+f(z) \quad \text { for any } z \in b D .
$$

We will show that the quantity

$$
\int_{w \in b D} \frac{f(w)-f(z)}{\Delta^{2}\left(w, z_{\epsilon}\right)} d \lambda(w)
$$

converges uniformly in $z \in b D$ as $\epsilon \rightarrow 0$. To this end, we consider the following smooth approximation of $\rho$, see [LS-1, Section 5]:

$$
\rho_{\gamma}(w)=\left(u_{1}^{2}+\gamma\right)^{m / 2}+v_{1}^{2}+u_{2}^{2}+v_{2}^{2}-1 .
$$

Then for any $0<\gamma<1$ we have

$$
\int_{w \in b D} \frac{f(w)-f(z)}{\Delta^{2}\left(w, z_{\epsilon}\right)} d \lambda(w)=A_{\gamma}\left(z_{\epsilon}\right)+B_{\gamma}\left(z_{\epsilon}\right)
$$

with

$$
A_{\gamma}\left(z_{\epsilon}\right)=-\frac{1}{4 \pi^{2}} \int \frac{f(w)-f(z)}{w \in b D} j^{*}\left(\partial \rho \wedge \bar{\partial} \partial\left(\rho-\rho_{\gamma}\right)\right)(w) .
$$

and

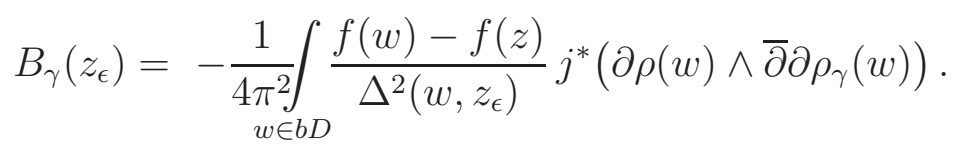

The dominated convergence theorem grants that

$$
A_{\gamma}\left(z_{\epsilon}\right) \rightarrow 0 \text { as } \gamma \rightarrow 0
$$


for each fixed $z_{\epsilon}$. Next we deal with the term $B_{\gamma}\left(z_{\epsilon}\right)$, again assuming that $z_{\epsilon}$ has been fixed. Applying Stokes' theorem to the manifold $M=$ $b D$ (which has $b M=\emptyset$ ) we obtain that

$$
0=\int_{w \in b D} d_{w} j^{*}\left(\frac{1}{\Delta\left(w, z_{\epsilon}\right)}(f(w)-f(z)) \bar{\partial} \partial \rho_{\gamma}(w)\right) .
$$

Thus (since $d \bar{\partial} \partial \rho_{\gamma} \equiv 0$ ) we obtain

$$
\begin{gathered}
0=\int_{w \in b D} \frac{1}{\Delta\left(w, z_{\epsilon}\right)} j^{*}\left(d f(w) \wedge \bar{\partial} \partial \rho_{\gamma}(w)\right)+ \\
-\int_{w \in b D} \frac{f(w)-f(z)}{\Delta^{2}\left(w, z_{\epsilon}\right)} j^{*}\left[d_{w}\left(\Delta\left(w, z_{\epsilon}\right)\right] \wedge \bar{\partial} \partial \rho_{\gamma}(w) .\right.
\end{gathered}
$$

But

$$
d_{w} \Delta\left(w, z_{\epsilon}\right)=\partial \rho(w)+\nabla^{2} \rho(w) \cdot(w-z)
$$

where $\nabla^{2} \rho(w) \cdot(w-z)$ is short-hand for the 1-form

$$
\sum_{j=1}^{2}\left(\frac{\partial^{2} \rho(w)}{\partial w_{j} \partial w_{1}} d w_{1}+\frac{\partial^{2} \rho(w)}{\partial w_{j} \partial w_{2}} d w_{2}\right)\left(w_{j}-z_{j}\right) .
$$

It follows that

$$
\begin{gathered}
B_{\gamma}\left(z_{\epsilon}\right)=\int_{w \in b D} \frac{j^{*}\left(d f(w) \wedge \bar{\partial} \partial \rho_{\gamma}(w)\right)}{\Delta\left(w, z_{\epsilon}\right)}+ \\
-\int_{w \in b D} \frac{f(w)-f(z)}{\Delta^{2}\left(w, z_{\epsilon}\right)} \nabla^{2} \rho(w) \cdot(w-z) \wedge j^{*} \bar{\partial} \partial \rho_{\gamma}(w) .
\end{gathered}
$$

Invoking the dominated convergence theorem one more time, we obtain

$$
\begin{gathered}
\lim _{\gamma \rightarrow 0} B_{\gamma}\left(z_{\epsilon}\right)=\int_{w \in b D} \frac{j^{*}(d f(w) \wedge \bar{\partial} \partial \rho(w))}{\Delta\left(w, z_{\epsilon}\right)}+ \\
-\int_{w \in b D} \frac{f(w)-f(z)}{\Delta^{2}\left(w, z_{\epsilon}\right)} \nabla^{2} \rho(w) \cdot(w-z) \wedge j^{*} \bar{\partial} \partial \rho(w) .
\end{gathered}
$$

Combining all of the above we conclude that

$$
\mathbf{C}(f)\left(z_{\epsilon}\right)-f(z)=\mathbf{E}(d f)\left(z_{\epsilon}\right)+\mathbf{R}(f)\left(z_{\epsilon}\right)
$$

where we have set

$$
\mathbf{E}(d f)\left(z_{\epsilon}\right)=\int_{w \in b D} \frac{d f(w) \wedge j^{*}(\bar{\partial} \partial \rho(w))}{\Delta\left(w, z_{\epsilon}\right)}
$$


and

$$
\mathbf{R}(f)\left(z_{\epsilon}\right)=-\int_{w \in b D} \frac{f(w)-f(z)}{\Delta^{2}\left(w, z_{\epsilon}\right)} \nabla^{2} \rho(w) \cdot(w-z) \wedge j^{*} \bar{\partial} \partial \rho(w) .
$$

Define

$$
\mathbf{E}(d f)(z):=\int_{w \in b D} \frac{d f(w) \wedge j^{*}(\bar{\partial} \partial \rho(w))}{\Delta(w, z)}, \quad z \in b D
$$

and

$$
\mathbf{R}(f)(z):=-\int_{w \in b D} \frac{f(w)-f(z)}{\Delta^{2}(w, z)} \nabla^{2} \rho(w) \cdot(w-z) \wedge j^{*} \bar{\partial} \partial \rho(w), z \in b D .
$$

We shall next see that for any $z \in b D$, each of $\mathbf{E}(f)(z)$ and $\mathbf{R}(f)(z)$ is an absolutely convergent integral, and this in turn will grant that

$$
|\mathbf{E}(d f)(z)|<\infty ; \quad|\mathbf{R}(f)(z)|<\infty \quad \text { for any } z \in b D .
$$

To prove these assertions we recall that

$$
\operatorname{Re} \Delta(w, z) \geq c|w-z|^{2} \quad \text { for any } z \in b D \quad \text { and } w \in b D
$$

by the convexity of $D$, and that

$$
j^{*}(\bar{\partial} \partial \rho(w))=O\left(\left|u_{1}\right|^{m-2}\right) \quad \text { and } \quad\left|\nabla^{2} \rho(w)\right|=O\left(\left|u_{1}\right|^{m-2}\right) \text {. }
$$

Thus, since $f$ is supported in a coordinate patch of the first kind, writing $x_{1}=\operatorname{Re} z_{1}$ we have

$$
|\mathbf{E}(d f)(z)| \leq C \int_{w \in b D} \frac{|d f(w)|\left|u_{1}\right|^{m-2}}{|w-z|^{2}} d \sigma(w) \leq C \iiint_{u_{1}^{2}+u_{2}^{2}+v_{2}^{2}<1} \frac{\left|u_{1}-x_{1}\right|^{m-2}}{u_{1}^{2}+u_{2}^{2}+v_{2}^{2}} d u_{1} d u_{2} d v_{2} .
$$

The desired finiteness of $\mathbf{E}(d f)(z)$ now follows from Lemma 6 below applied with $\beta=0$ and $\alpha:=m-1 \in(0,1)$ (in fact our hypothesis that $3 / 2<m<2$ gives that $\alpha$ is in $(1 / 2,1))$.

Similarly we have, by (29), (31) and the fact that $f(w)-f(z)=$ $O(|w-z|)$, that 


$$
|\mathbf{R}(f)(z)| \leq C \int_{w \in b D} \frac{|f(w)|\left|u_{1}\right|^{2 m-4}}{|w-z|^{2}} d \sigma(w) \leq C \iiint_{u_{1}^{2}+u_{2}^{2}+v_{2}^{2}<1} \frac{\left|u_{1}-x_{1}\right|^{2 m-4}}{u_{1}^{2}+u_{2}^{2}+v_{2}^{2}} d u_{1} d u_{2} d v_{2} .
$$

The finiteness of $\mathbf{R}(f)(z)$ is again a consequence of Lemma 6 below applied with $\beta=0$ and $\alpha:=2 m-3$ (which is in $(0,1)$ thanks to our assumption that $3 / 2<m<2$ ).

To conclude the proof of the proposition we are left to show that of $\mathbf{E}(d f)\left(z_{\epsilon}\right)$ and $\mathbf{R}(f)\left(z_{\epsilon}\right)$ converge respectively to $\mathbf{E}(d f)(z)$ and $\mathbf{R}(f)(z)$ uniformly in $z \in b D$ (in fact, absolutely and uniformly in $z \in b D$ ). To prove the convergence of $\mathbf{E}(d f)\left(z_{\epsilon}\right)$, note that

$$
\left|\mathbf{E}(d f)\left(z_{\epsilon}\right)-\mathbf{E}(d f)(z)\right| \leq C \int_{w \in b D}\left|u_{1}\right|^{m-2}\left|\frac{1}{\Delta\left(w, z_{\epsilon}\right)}-\frac{1}{\Delta(w, z)}\right| d \sigma(w) .
$$

Now by the basic estimate for $\Delta\left(w, z_{\epsilon}\right)$ we have that

$$
\left|\frac{1}{\Delta\left(w, z_{\epsilon}\right)}-\frac{1}{\Delta(w, z)}\right| \leq \frac{c_{0} \epsilon}{\operatorname{Re} \Delta\left(w, z_{\epsilon}\right) \operatorname{Re} \Delta(w, z)}
$$

And since the basic estimate for $\operatorname{Re} \Delta\left(w, z_{\epsilon}\right)$ in particular gives $\epsilon \lesssim$ $\operatorname{Re} \Delta\left(w, z_{\epsilon}\right)$, we also have that

$$
\epsilon^{1-\beta} \lesssim \operatorname{Re} \Delta\left(w, z_{\epsilon}\right)^{1-\beta} \quad \text { for any } 0 \leq \beta<1 .
$$

Inserting this in the above (and using once again the basic estimate: $\left.\operatorname{Re} \Delta(w, z) \lesssim \operatorname{Re} \Delta\left(w, z_{\epsilon}\right)\right)$ we obtain

$$
\left|\mathbf{E}(f)\left(z_{\epsilon}\right)-\mathbf{E}(f)(z)\right| \leq C \epsilon^{\beta} \int_{w \in b D} \frac{\left|u_{1}\right|^{m-2}}{\operatorname{Re} \Delta(w, z)^{1+\beta}} d \sigma(w) .
$$

Invoking one more time the convexity of $D$ we conclude that the above integral is further bounded by

$$
C \epsilon^{\beta} \int \frac{\left|u_{1}\right|^{m-2}}{|w-z|^{2+2 \beta}} d \sigma(w) \leq C \epsilon^{\beta} \iiint_{u_{1}^{2}+u_{2}^{2}+v_{2}^{2}<1} \frac{\left|u_{1}-x_{1}\right|^{m-2}}{\left(u_{1}^{2}+u_{2}^{2}+v_{2}^{2}\right)^{1+\beta}} d u_{1} d u_{2} d v_{2} .
$$

The desired conclusion now follows by Lemma [ with $\alpha=m-1$ and with any $0<\beta<(m-1) / 2$.

Finally, we claim that

$$
\mathbf{R}(f)\left(z_{\epsilon}\right)-\mathbf{R}(f)(z) \rightarrow 0 \quad \text { as } \quad \epsilon \rightarrow 0
$$


uniformly in $z \in b D$. To see this we begin as before, with

$$
\left|\mathbf{R}(f)\left(z_{\epsilon}\right)-\mathbf{R}(f)(z)\right| \leq C \int_{w \in b D}\left|u_{1}\right|^{2 m-4}|w-z|^{2}\left|\frac{1}{\Delta^{2}\left(w, z_{\epsilon}\right)}-\frac{1}{\Delta^{2}(w, z)}\right| d \sigma(w) .
$$

By the basic estimate for $\Delta(w, z)$ we see that

$$
\begin{gathered}
\left|\frac{1}{\Delta^{2}\left(w, z_{\epsilon}\right)}-\frac{1}{\Delta^{2}(w, z)}\right| \leq \frac{c_{1} \epsilon\left|\Delta\left(w, z_{\epsilon}\right)\right|}{\left|\Delta^{2}\left(w, z_{\epsilon}\right) \Delta^{2}(w, z)\right|}+\frac{c_{1} \epsilon|\Delta(w, z)|}{\left|\Delta^{2}\left(w, z_{\epsilon}\right) \Delta^{2}(w, z)\right|} \leq \\
\leq \frac{c_{1} \epsilon}{\operatorname{Re} \Delta\left(w, z_{\epsilon}\right)(\operatorname{Re} \Delta(w, z))^{2}}+\frac{c_{1} \epsilon}{\left(\operatorname{Re} \Delta\left(w, z_{\epsilon}\right)\right)^{2} \operatorname{Re} \Delta(w, z)} \leq \\
\leq \frac{2 c_{1} \epsilon}{\operatorname{Re} \Delta\left(w, z_{\epsilon}\right)(\operatorname{Re} \Delta(w, z))^{2}}=\frac{2 c_{1} \epsilon^{\beta} \epsilon^{1-\beta}}{\operatorname{Re} \Delta\left(w, z_{\epsilon}\right)(\operatorname{Re} \Delta(w, z))^{2}} .
\end{gathered}
$$

Using again the trick: $\epsilon^{1-\beta} \leq\left(\operatorname{Re} \Delta\left(w, z_{\epsilon}\right)\right)^{1-\beta}$ for any $0 \leq \beta<1$, we bound the latter with

$$
\frac{2 c_{1} \epsilon^{\beta}}{\left(\operatorname{Re} \Delta\left(w, z_{\epsilon}\right)\right)^{\beta}(\operatorname{Re} \Delta(w, z))^{2}} \leq \frac{2 c_{1} \epsilon^{\beta}}{(\operatorname{Re} \Delta(w, z))^{2+\beta}}
$$

Finally, by the convexity of $D$ we conclude that

$$
\left|\frac{1}{\Delta^{2}\left(w, z_{\epsilon}\right)}-\frac{1}{\Delta^{2}(w, z)}\right| \leq C \frac{\epsilon^{\beta}}{|w-z|^{4+2 \beta}} .
$$

Combining all of the above we conclude that

$$
\begin{gathered}
\left|\mathbf{R}(f)\left(z_{\epsilon}\right)-\mathbf{R}(f)(z)\right| \leq C \epsilon^{\beta} \int \frac{\left|u_{1}\right|^{2 m-4}}{|w-z|^{2+2 \beta}} d \sigma(w) \leq \\
\leq C \epsilon^{\beta} \iiint_{u_{1}^{2}+u_{2}^{2}+v_{2}^{2}<1} \frac{\left|u_{1}-x_{1}\right|^{2 m-4}}{\left(u_{1}^{2}+u_{2}^{2}+v_{2}^{2}\right)^{1+\beta}} d u_{1} d u_{2} d v_{2} .
\end{gathered}
$$

The desired conclusion now follows by applying Lemma 6 with $\alpha=$ $2 m-3$ (which is in $(0,1)$ thanks to our hypothesis that $3 / 2<m<2$ ) and with any $0<\beta<\alpha / 2=m-3 / 2$.

This concludes the proof of the Proposition (assuming the truth of Lemma 6, whose proof is given below). 
Lemma 6. Suppose that $\left|x_{1}\right| \leq 1$. Then we have that

$$
I_{\alpha, \beta}:=\iiint_{u_{1}^{2}+u_{2}^{2}+v_{2}^{2}<1} \frac{\left|u_{1}-x_{1}\right|^{-1+\alpha}}{\left(u_{1}^{2}+u_{2}^{2}+v_{2}^{2}\right)^{1+\beta}} d u_{1} d u_{2} d v_{2} \leq A_{\alpha, \beta}<\infty
$$

is true for any $\alpha \in(0,1]$ and any $0 \leq \beta<\alpha / 2$. The constant $A_{\alpha, \beta}$ is independent of $x_{1}$.

Proof. Note that $I_{\alpha, 0} \leq I_{\alpha, \beta}$ for any $\beta>0$, thus we only need prove the conclusion in the case when $0<\beta<\alpha / 2$.

To begin with, we write

$$
\begin{gathered}
\iiint_{u_{1}^{2}+u_{2}^{2}+v_{2}^{2}<1} \frac{\left|u_{1}-x_{1}\right|^{-1+\alpha}}{\left(u_{1}^{2}+u_{2}^{2}+v_{2}^{2}\right)^{1+\beta}} d u_{1} d u_{2} d v_{2} \leq \\
=\int_{\left|u_{1}\right| \leq 2}\left|u_{1}-x_{1}\right|^{-1+\alpha}\left(\iint_{\mathbb{R}^{2}} \frac{1}{\left(u_{1}^{2}+u_{2}^{2}+v_{2}^{2}\right)^{1+\beta}} d u_{2} d v_{2}\right) d u_{1} .
\end{gathered}
$$

Notice that the change of variables: $u_{2}:=\left|u_{1}\right| \widetilde{u}_{2}$ and $v_{2}:=\left|u_{1}\right| \widetilde{v}_{2}$, gives

$\iint_{\mathbb{R}^{2}} \frac{1}{\left(u_{1}^{2}+u_{2}^{2}+v_{2}^{2}\right)^{1+\beta}} d u_{2} d v_{2}=\left|u_{1}\right|^{-2 \beta} \iint_{\mathbb{R}^{2}} \frac{1}{\left(1+\widetilde{u}_{2}^{2}+\widetilde{v}_{2}^{2}\right)^{1+\beta}} d \widetilde{u}_{2} d \widetilde{v}_{2} \leq c\left|u_{1}\right|^{-2 \beta}$

with $c=c(\beta)$ (in particular $C$ is independent of $u_{1}$ ). Thus

$$
I_{\alpha, \beta} \leq c \int_{\left|u_{1}\right| \leq 2}\left|u_{1}\right|^{-2 \beta}\left|u_{1}-x_{1}\right|^{-1+\alpha} d u_{1}=I+I I+I I I,
$$

where we have set

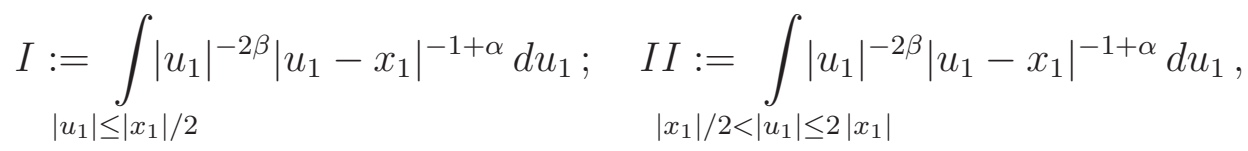

and

$$
I I I:=\int_{\left|u_{1}\right|>2\left|x_{1}\right|}\left|u_{1}\right|^{-2 \beta}\left|u_{1}-x_{1}\right|^{-1+\alpha} d u_{1}
$$


Now the active integrand factor for $A$ is $\left|u_{1}\right|^{-2 \beta}$, that is

$$
I \leq\left|x_{1}\right|^{-1+\alpha} \int_{\left|u_{1}\right| \leq\left|x_{1}\right| / 2}\left|u_{1}\right|^{-2 \beta} d u_{1}=a\left|x_{1}\right|^{-1+\alpha}\left|x_{1}\right|^{1-2 \beta}=a\left|x_{1}\right|^{\alpha-2 \beta}=O(1)
$$

since $\alpha>2 \beta$ (and $a=a(\beta)$, that is $a$ is independent of $x_{1}$ ).

On the other hand, the active integrand factor in $I I$ is $\left|u_{1}-x_{1}\right|^{-1+\beta}$, that is

$$
I I \leq\left|x_{1}\right|^{-2 \beta} \int_{\left|x_{1}\right| / 2<\left|u_{1}\right| \leq 2\left|x_{1}\right|}\left|u_{1}-x_{1}\right|^{-1+\alpha} d u_{1} \leq\left|x_{1}\right|^{-2 \beta} \int_{\left|u_{1}\right| \leq 3\left|x_{1}\right|}\left|u_{1}\right|^{-1+\alpha} d u_{1}=b\left|x_{1}\right|^{-2 \beta+\alpha}=O(1)
$$

(again, here $b=b(\alpha)$ is independent of $x_{1}$ ).

Finally

$$
I I I \leq \int_{\left|u_{1}\right|>2\left|x_{1}\right|}\left|u_{1}\right|^{-2 \beta}\left|u_{1}\right|^{-1+\alpha} d u_{1} \leq \int_{0}^{\infty}\left|u_{1}\right|^{-1+\alpha-2 \beta} d u_{1}=c<\infty
$$

again because $\alpha-2 \beta \in(0,1)$ (and with $c=c(\alpha, \beta)$ independent of $\left.x_{1}\right)$.

While we are unable to prove that the Cauchy-Leray integral for the domain (33) extends to a continuous function on the entire closure $\bar{D}$ when $1<m \leq 3 / 2$, we have the following result, valid for each $1<m<2$, whose proof will appear elsewhere.

Proposition 7. Suppose that $f \in C^{1}(b D)$. Then $\mathbf{C}(f)$ extends to a continuous function in $\bar{D} \backslash\left\{b D \cap\left\{x_{1}=0\right\}\right\}$. More precisely, for $z \in b D$ set

$$
z_{\epsilon}=z+\epsilon \nu(z) \in D
$$

Then, we have that $\mathbf{C}(f)\left(z_{\epsilon}\right)$ converges uniformly in $z \in b D \backslash\left\{x_{1}=\right.$ $0\}$ to a limit that we denote $\mathcal{C}(f)(z)$ which satisfies

$$
\begin{gathered}
|\mathcal{C}(f)(z)| \leq C\left|x_{1}\right|^{-2+m} . \\
\text { REFERENCES }
\end{gathered}
$$

\section{REFERENCES}

[APS] Andersson M., Passare M. and Sigurdsson R., Complex convexity and analytic functionals, Birkhäuser, Basel (2004).

[BaLa] Barrett D. and Lanzani L., The Leray transform on weighted boundary spaces for convex Reinhardt domains, J. Funct. Analysis, 257 (2009), 27802819.

[C] Calderòn A. P, Cauchy integrals on Lipschitz curves and related operators, Proc. Nat. Acad. Sci. 74 no. 4, (1977) 1324-1327. 
[CMM] R. Coifman, A. McIntosh and Y. Meyer, L'intègrale de Cauchy dèfinit un opèrateur bornè sur $L^{2}$ pour les courbes lipschitziennes Ann. of Math. 116 (1982) no. 2, 361 - 387.

[Da] G. David, Opérateurs intégraux singuliers sur certain courbes du plan complèxe, Ann. Scient. Éc. Norm. Sup. 17 (1984), 157-189.

[Hans] Hansson T., On Hardy spaces in complex ellipsoids, Ann. Inst. Fourier (Grenoble) 49 (1999), 1477-1501.

[Hö] Hörmander, L. Notions of convexity, Birkhäuser, Basel (1994).

$[\mathrm{K}] \quad$ Krantz S., Canonical kernels versus constructible kernels, preprint. ArXiv: 1112.1094 .

[LS-1] Lanzani L. and Stein E. M. The Cauchy-Leray integral: counterexamples to the $L^{p}$-theory, preprint, arXiv: 1701.03812.

[LS-2] Lanzani L. and Stein E. M. The Cauchy integral in $\mathbb{C}^{n}$ for domains with minimal smoothness, Advances in Math. 264 (2014) 776-830, DOI: dx.doi.org/10.1016/j.aim.2014.07.016).

[LS-3] Lanzani L. and Stein E. M. Cauchy-type integrals in several complex variables, Bull. Math. Sci. 3 (2) (2013), 241-285, DOI: 10.1007/s13373-0130038-y.

[Ra] Range M., Holomorphic functions and integral representations in several complex variables, Springer Verlag, Berlin, (1986).

[Ro-1] Rotkevich A. S., The Cauchy-Leray-Fantappi integral in linearly convex domains (Russian) Zap. Nauchn. Sem. S.-Peterburg. Otdel. Mat. Inst. Steklov. (POMI) 401 (2012), Issledovaniya po Lineinym Operatoram i Teorii Funktsii 40, 172-188, 201; translation in J. Math. Sci. (N. Y.) 194 (2013), no. 6, 693-702.

Dept. of Mathematics, Syracuse University Syracuse, Ny 132441150 USA

E-mail address: 1lanzani@syr.edu

Dept. of Mathematics, Princeton University, Princeton, NJ 08544100 USA

E-mail address: stein@math.princeton.edu 